\title{
MEASUREMENT AND MODELING OF MAGNETIC CONFIGURATIONS TO MIMIC OVERLOAD SCENARIOS IN THE W7-X STELLARATOR
}

\author{
J.D. Lore ${ }^{1}$, Y. Gao ${ }^{2}$, J. Geiger ${ }^{2}$, H. Hoelbe ${ }^{2}$, H. Niemann ${ }^{2}$, M. Jakubowski², G.A. Wurden ${ }^{3}$, S. Lazerson ${ }^{4}$, \\ P. Drewelow ${ }^{2}$, A. Ali ${ }^{2}$, A. Puig Sitjes ${ }^{2}$, A. LeViness ${ }^{5}$, H. Frerichs ${ }^{6}$, T. Barbui ${ }^{6}$, F. Effenberg 6 , J. Harris ${ }^{1}$, A.

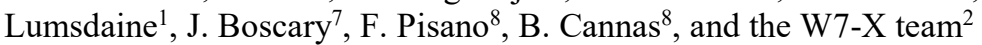

1 Oak Ridge National Laboratory, Oak Ridge, TN, USA

2 Max Planck Institute for Plasma Physics, Greifswald, Germany

3 Los Alamos National Laboratory, Los Alamos, NM, USA

4 Princeton Plasma Physics Laboratory, Princeton, NJ, USA

5 Princeton University, Princeton, NJ, USA

6 University of Wisconsin-Madison, Madison, WI, USA

7 Max Planck Institute for Plasma Physics, Garching, Germany

8 University of Cagliari, Cagliari, Italy

\begin{abstract}
Experiments were performed during the first divertor operational phase (OP1.2a) of the Wendelstein 7-X stellarator to verify predictions of potential overload conditions corresponding to certain high-power long-pulse OP2 scenarios. A potential solution to this overload is the installation of new divertor components called scraper elements, which are designed to intercept heat flux that would otherwise be incident on low-rated divertor edges. Heat flux measurements were obtained in a series of magnetic configurations designed to mimic the magnetic topology evolution caused by net toroidal current and beta, which is not directly accessible in OP1.2. The experimental flux patterns are qualitatively reproduced in position and magnitude for by field line diffusion simulations using ad-hoc cross-field diffusivities near the value used to design the scraper element. However, some important differences are observed, including a shift towards the pumping gap and lowrated components. Potential sources of discrepancy such as toroidal current evolution and error fields are discussed. A shift in the experimental heat flux pattern due to increasing toroidal current is observed in a 12 second discharge.
\end{abstract}

\section{INTRODUCTION}

The 'scraper element' is a component of the Wendelstein 7-X (W7-X) divertor intended to protect other components from overload in certain scenarios with significant toroidal current $[1,2,3]$. The overload scenario is predicted to occur in the upcoming operational phase 2 (OP2, scheduled to begin in 2020), in which activelycooled divertor components will allow for pulse lengths up to 30 minutes with $10 \mathrm{MW}$ of input power. Under these conditions, transport simulations of the standard configuration (with an edge 5/5 island chain) with 7 MW of input power yield a steady-state toroidal current of $\sim 40 \mathrm{kA}$, which evolves over $\sim 100$ seconds [4] and changes the topology of the magnetic field, primarily by increasing the rotational transform at the plasma edge. The exhaust solution in $\mathrm{W} 7-\mathrm{X}$ is an island divertor where the edge island chain is intersected by the divertor components, ideally yielding helical 'stripes' of deposited heat and particle fluxes on the divertor allowing for power removal and efficient neutral pumping. Increasing the edge transform causes the $5 / 5$ island to move inwards towards the axis, shifting the deposited flux patterns and ultimately resulting in a limited configuration. In addition, plasma pressure $(<\beta>\sim 3 \%$ in this scenario) causes the island width to increase and the Shafranov shift can cause low-rated baffle components to receive a convective load. To compensate for these effects, a new magnetic configuration was developed with a reduced vacuum transform and an inward shift which then results in the desired edge transform and reduced baffle loads in steady-state [4]. However, at intermediate levels of net toroidal current ( 22-32 kA) the edges of the 'horizontal target' (and to a lesser degree the 'vertical target') are predicted to receive fluxes in excess of the $5 \mathrm{MW} / \mathrm{m}^{2}$ rating [5]. A poloidal shift of the strike lines due to evolving toroidal current has already been observed in W7-X [6].

\footnotetext{
1 This manuscript has been authored by UT-Battelle, LLC, under contract DE-AC05-00OR22725 with the US Department of Energy (DOE). The US government retains and the publisher, by accepting the article for publication, acknowledges that the US government retains a nonexclusive, paid-up, irrevocable, worldwide license to publish or reproduce the published form of this manuscript, or allow others to do so, for US government purposes. DOE will provide public access to these results of federally sponsored research in accordance with the DOE Public Access Plan (http://energy.gov/downloads/doepublic-access-plan).
} 
Figure 1 shows the main components of a W7-X divertor module. The horizontal and vertical targets, as well as the 'high iota tail' are the primary divertor components, which are designed to withstand peak heat fluxes of $10 \mathrm{MW} / \mathrm{m}^{2}$ over much of the surface area during water-cooled operation (OP2) [5]. The targets are labeled according to their orientation in the bean-shaped $\left(\varphi=0^{\circ}\right)$ plane, and do not correspond to the tokamak divertor naming convention [7]. The overload cases occur at approximately 40 seconds into the scenario as the magnetic topology transitions from limited to island diverted, and the target edges are wetted. The scraper element was designed as a passive solution to this issue by intercepting flux to the overloaded areas, without exceeding its own $20 \mathrm{MW} / \mathrm{m}^{2}$ rating. This OP2 scenario with predicted overload is referred to as the scraper element reference scenario (SE-RS). Other potential solutions to this scenario include active control of edge transform using the planar coil set, electron cyclotron current drive (ECCD) to counteract the bootstrap current, and ECCD to accelerate the evolution while the injected power is low [8,9]. The transport simulations used to determine the SE-RS use a relatively simple model for anomalous transport [9], which must be validated against W7-X experimental data. Deviations from the assumed scaling will modify the predicted pressure and bootstrap current profiles.

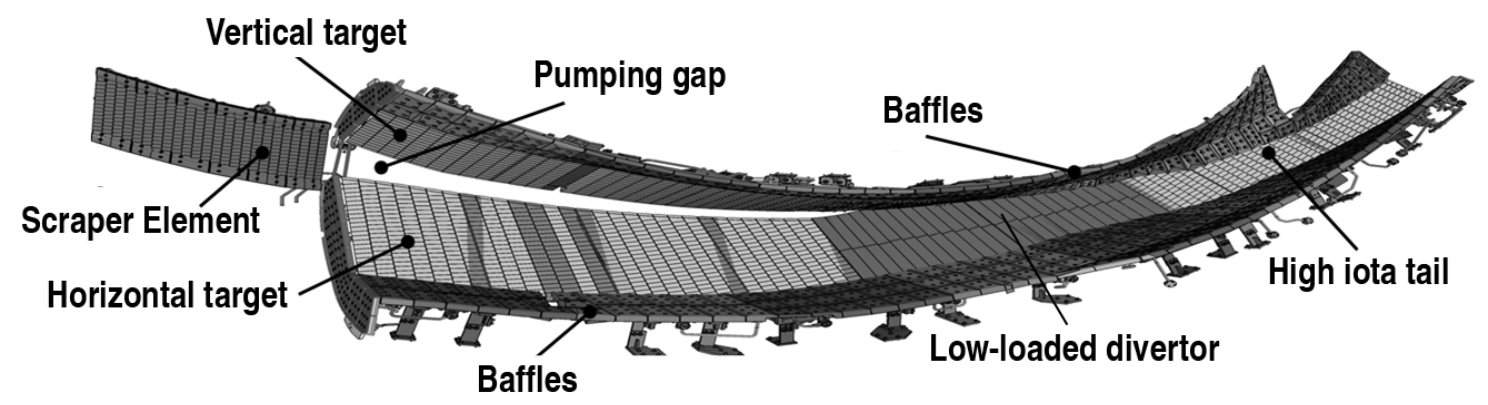

FIG. 1. Components in one of ten W7-X divertor modules including the scraper element, figure from [1].

The introduction of a new divertor component to W7-X yields potential deleterious effects and significant challenges, not the least of which is the cost of its design, manufacturing, and installation. The scraper element was designed after the other divertor components were finalized, and the physics and engineering optimization procedure [1] included avoiding changes to the existing divertor, baffle, and vessel protection elements. Ideally the scraper would only receive flux during the overload portion of the discharge, however this would require significant poloidal curvature of the element. This was not possible due to the cooled twisted-tape monoblock design and interference with the vessel protection elements. As a result of these constraints, the scraper element receives flux in the steady-state SE-RS after the current has evolved beyond the point where heat flux could damage other components.

Any new wetted component results in an additional source of recycled and sputtered particles. However, the scraper element is in an unbaffled region such that recycled neutrals are not preferentially directed towards the pump. The removal of flux from the target edges in steady-state further reduces the neutral flux into the pumping gap. The low rating on the edge of the horizontal and vertical targets (where the cooling channel makes a U-bend [10]) exacerbates the pumping problem, as wetting the area directly adjacent to the pump should maximize the plenum pressure [11]. This makes the relative decrease in the pumping efficiency due to the scraper element ( $\sim 50 \%$ during overload and $\sim 25 \%$ in steady-state [12]) seem especially large, however the reference plenum pressures should be compared to a derated SE-RS that does not cause overload.

Experiments are required to assess the key physics aspects of the scraper element [2]. This includes protection of the target edges, the load onto the scraper element, lack of interference with other reference scenarios, and the impact on fuelling and pumping. The SE-RS is not directly accessible in the early operational phases of W7-X (OP1.2) due to limits on the injected power and pulse length. To overcome this, a set of new magnetic configurations were developed to approximately reproduce the important features in the deposited flux to the target edges and the scraper element [13]. These 'mimic configurations' use the planar coil set to control the edge transform and the control coils to adjust the edge island size, to mimic the effect of a net toroidal current and plasma beta, respectively. Five mimic configurations were developed corresponding to different time points in the SE-RS, which are labelled by the net toroidal current: $\{0,11,22,32,43\} \mathrm{kA}$. The same five time points were also used in the design of the scraper element [1]. Experiments were performed in each mimic configuration in OP1.2a to test the approach of mimicking OP2 scenarios using low-beta configurations, and 
verify that the heat loads were similar to the predictions and sufficient for testing the scraper elements in OP1.2b.

The remainder of the paper describes results from OP1.2a and is organized as follows. The experimental setup of the mimic configurations and an analysis of the measured heat flux data is given in Section 2 . The experimental results are compared to field line diffusion calculations in Section 3. Sources of a poloidal shift in the heat flux profiles are discussed in section 4. Finally, conclusions are given in Section 5.

\section{EXPERIMENTAL SETUP}

Experiments were performed during the first portion of OP1.2 without any scraper elements installed (OP1.2a). The goal of these experiments was to obtain data in each of the five mimic magnetic configurations to compare the measured flux patterns to predictions and validate the approach of using the W7-X coil set to mimic otherwise inaccessible OP2-like conditions. Evaluation of scraper element performance requires assessment of heat fluxes and neutral pressures, however a technical issue with neutral gas manometers during OP1.2a, as well as the significant change in plasma conditions due to OP1.2b boronization prevent a direct comparison of neutral pressures between the campaigns. For this reason, we focus the analysis of OP1.2a data on the heat flux comparison to simulation. Two test divertor unit (TDU, not actively cooled) scraper elements were installed before OP1.2b, allowing for experiments to confirm the target edge protection and assess the impact of the scraper element on fuelling and pumping. The impact of the scraper elements on neutral transport and pumping will be evaluated by comparing modules with and without TDU scraper elements. Results from OP1.2b will be presented in a future publication.

The experiment performed during OP1.2a operated in the five mimic configurations, as described in Table 1. The winding currents are listed in amps for the modular coils $\left(\mathrm{I}_{1}-\mathrm{I}_{5}, 108\right.$ windings), planar coils $\left(\mathrm{I}_{\mathrm{A}}, \mathrm{I}_{\mathrm{B}}\right.$, 36 windings), and control coils ( $\mathrm{I}_{\mathrm{C} 1}, \mathrm{I}_{\mathrm{C} 2}, 8$ windings). The trim coil set was not used, and the polarity of the control coils is such that the edge island size is increased in a stellarator symmetric manner. The 0kA and 43kA configurations are the OP2 SE-RS startup and steady-state cases, respectively. Both the 22kA and $32 \mathrm{kA}$ configurations are predicted to result in overload of the target edges.

TABLE 1. COIL CURRENTS FOR THE MIMIC CONFIGURATIONS USED IN OP1.2A.

\begin{tabular}{lcccccccccc}
\hline Label & Configuration & $\mathrm{I}_{1}$ & $\mathrm{I}_{2}$ & $\mathrm{I}_{3}$ & $\mathrm{I}_{4}$ & $\mathrm{I}_{5}$ & $\mathrm{I}_{\mathrm{A}}$ & $\mathrm{I}_{\mathrm{B}}$ & $\mathrm{I}_{\mathrm{C} 1}$ & $\mathrm{I}_{\mathrm{C} 2}$ \\
\hline $0 \mathrm{kA}$ & $\mathrm{EES}+252$ & 12022 & 11897 & 12148 & 13399 & 13524 & 8212 & -3005 & 2500 & -2500 \\
$11 \mathrm{kA}$ & EFS+252 & 12129 & 12002 & 12255 & 13519 & 13645 & 7391 & -3980 & 2500 & -2500 \\
$22 \mathrm{kA}$ & $\mathrm{EGS}+252$ & 12243 & 12116 & 12371 & 13646 & 13774 & 6504 & -4973 & 2500 & -2500 \\
$32 \mathrm{kA}$ & EGS001+252 & 12359 & 12230 & 12487 & 13775 & 13904 & 5600 & -5987 & 2500 & -2500 \\
$43 \mathrm{kA}$ & $\mathrm{FHS}+252$ & 12477 & 12347 & 12607 & 13907 & 14037 & 4679 & -7019 & 2500 & -2500 \\
\hline
\end{tabular}

Figure 2 shows Poincare plots and plasma facing components at a toroidal angle of $\varphi=1.75^{\circ}$, which is the approximate location of the predicted large edge overload in the $22 \mathrm{kA}$ configuration, for the mimic configurations (a-e) and the corresponding OP2 SE-RS configurations (f-j). The field lines are followed in vacuum for the mimic configurations, while in the SE-RS cases the magnetic field is calculated from VMEC [14,15] and EXTENDER [16] using the 'alternate' formulation (see Refs. [2,4,16] for discussion of the field extension and the impact on heat transport simulations). The mimic configurations are qualitatively different in the shape of the 5/5 island chain, however the position of the island relative to the target plates (and the scraper element) produces similar flux patterns as compared to the SE-RS configurations [13]. 

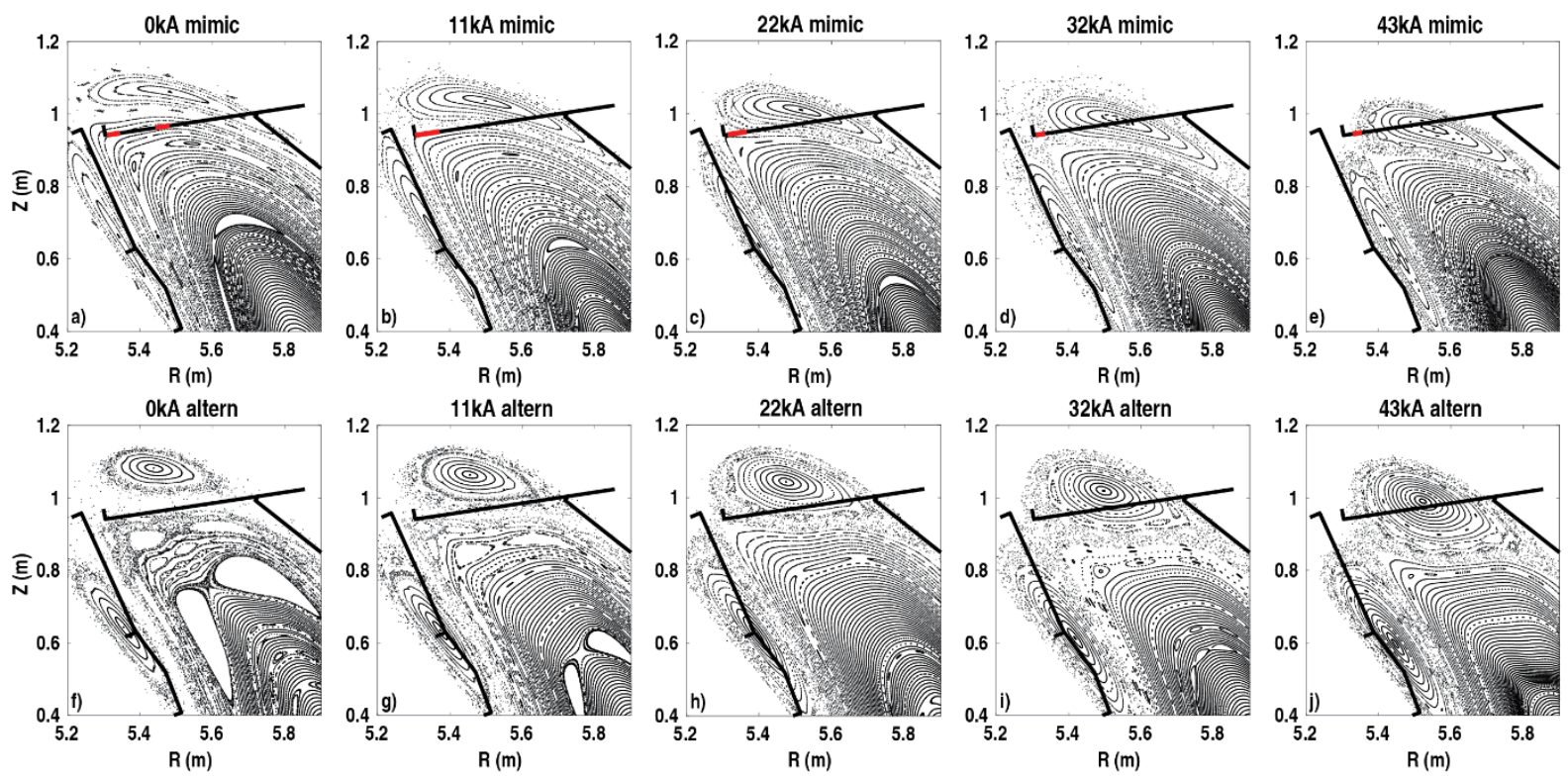

FIG. 2. Poincare plots at $\varphi=1.75^{\circ}$ for the mimic configurations (a-e) corresponding to the OP2 SE-RS configurations $(f-j)$. Red lines in (a-e) indicate position on horizontal target (defined by half-width) of experimental heat flux.

Figure 3 shows the time history of a hydrogen plasma with 2 MW of ECRH input power in each mimic configuration. The target average density $\int n_{e} d l / \int d l$ was $1.2 \cdot 10^{19} \mathrm{~m}^{-3}$, although some variation due to intermittent gas puff and varying wall conditions can be seen. The OP1.2a campaign largely used helium 'cleanup' plasmas for wall conditioning; boronization in OP1.2b has resulted in improved conditions.

Figure 4 shows experimental heat fluxes as measured via infrared cameras taken from the time window 3000-3100 ms. Unless otherwise noted, the data is from a camera viewing the upper divertor of module four, which was considered to be representative of 10 camera systems. The camera images are mapped to a scene model [17-20] for the horizontal target, vertical target, and low-loaded divertor components (the latter is a low rated divertor component sometimes labeled as 'bafhormid' that connects the horizontal target and the high iota tail, see Fig. 1). The flux patterns on the horizontal target show the transition from a limited (wide 'blob') to an island diverted topology (well defined 'stripe') as the configuration is changed from 0kA to 43kA (over a series of discharges). At $22 \mathrm{kA}$ the peak heat flux is on the very edge of the horizontal target and can also be observed on shielding components inside the pumping gap (not shown).

This is consistent with predictions of fluxes onto the sides of the horizontal target inside the pumping gap by DIV3D [1], which are particularly undesirable due to the low rating of this region and the higher incident angle of the magnetic field. The $32 \mathrm{kA}$ configuration is also an overload scenario for OP2 components, where the wetted area on the horizontal target is on the low rated region.
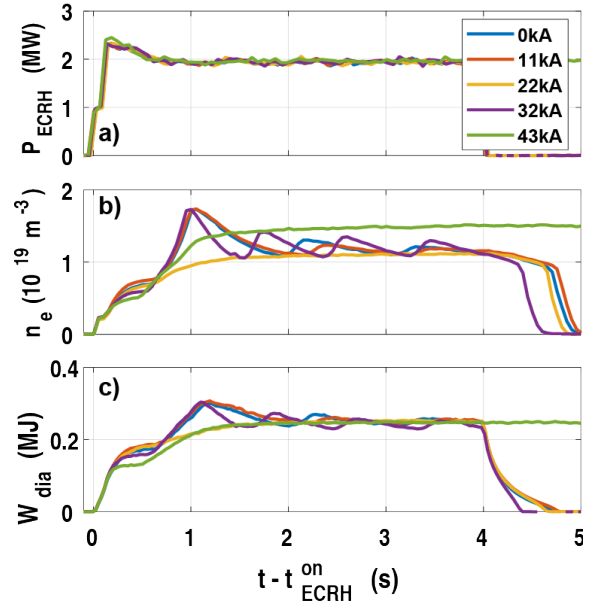

FIG. 3. Time history of a) input power, $b$ ) average electron density, and c) stored energy for a hydrogen plasma in each mimic magnetic configuration. 


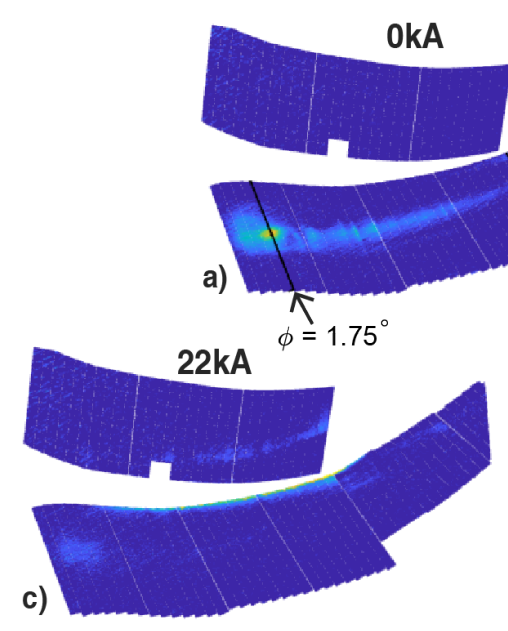

OkA
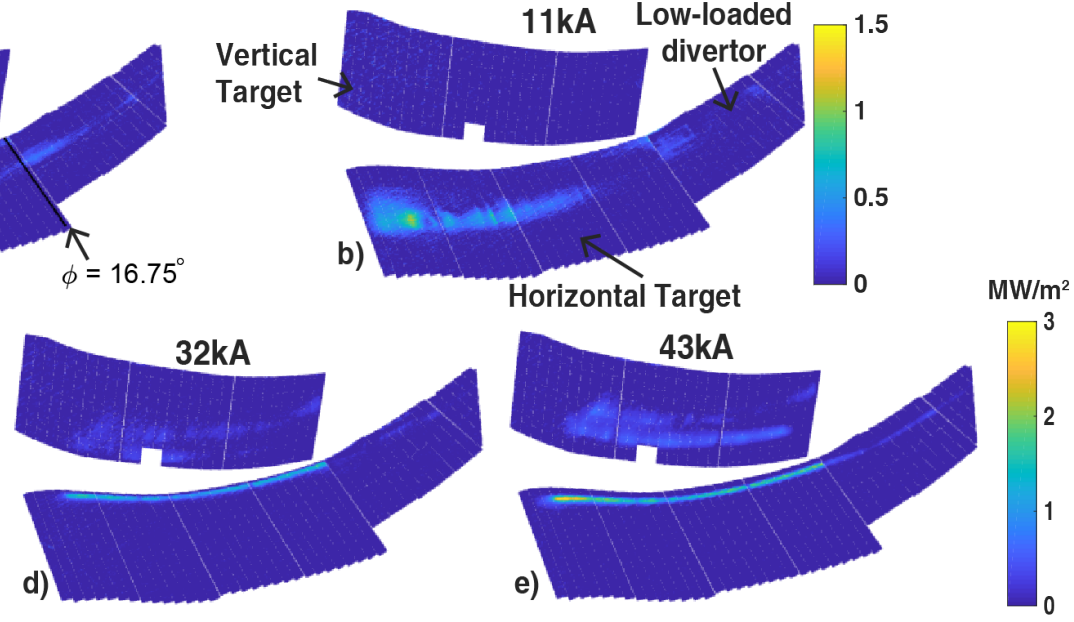

FIG. 4. Experimental heat fluxes to the vertical and horizontal targets and low-loaded divertor in a) $0 \mathrm{kA}$, b) $11 \mathrm{kA}$ [upper colorbar], c) $22 \mathrm{kA}, d$ ) $32 \mathrm{kA}$, and e) $43 \mathrm{kA}$ [lower color bar] mimic configurations with $2 \mathrm{MW}$ of input power.

Two toroidal angles of interest are labeled and indicated by black lines in Fig. 4a. The maximum edge load in the $22 \mathrm{kA}$ configuration (Fig. $4 \mathrm{c}$ ) occurs near $\varphi=1.75^{\circ}$, while $\varphi=16.75^{\circ}$ is the position of the prominent flux feature at 0kA (Fig. 4a). Radial profiles of heat flux at these toroidal angles are shown in Figure 5. For each experimental profile the position on the horizontal target of the peak heat flux has been marked in the Poincare plots in Figure $1\left(\varphi=1.75^{\circ}\right)$ and Figure $6\left(\varphi=16.75^{\circ}\right)$ by a red line. The line indicates the half-width of the heat flux. From this information the flux patterns in Fig. 4 can be largely understood. At $\varphi=1.75^{\circ}$ the $0 \mathrm{kA}$ configuration has two small peaks on the horizontal target (both shown in Fig. 2a) on either side of the 5/5 island chain as the field angle of the last closed flux surface (LCFS) is very grazing. The LCFS is defined by either the first closed surface intercepting a component, or the last field line that traces out a 2D line in the Poincare plot. The vertical target is not wetted as it intersects closed surfaces beyond the LCFS. At $\varphi=16.75^{\circ}$ the angle in the poloidal plane is greater, and a single peak of higher flux is observed. As the $5 / 5$ island chain moves inward with increasing edge transform (larger mimicked toroidal current) the $x$-point of the $5 / 5$ island chain approaches the edge of the horizontal target at $\varphi=1.75^{\circ}$ resulting in a large flux. At the same time, the flux at $\varphi=16.75^{\circ}$ decreases as the distance to the LFCS increases. As will be discussed below, at 22kA the flux at $\varphi=16.75^{\circ}$ can be expected to increase with increasing cross-field transport as there is not a direct intersection with the LCFS (or the near-LCFS island separatrix remnants) and the region is only wetted via cross-field diffusion. Finally, in the $32 \mathrm{kA}$ and $43 \mathrm{kA}$ configurations the island chain has the proper position relative to the target plate and the stripe pattern is formed by open field lines with heat diffusion through the x-point region.
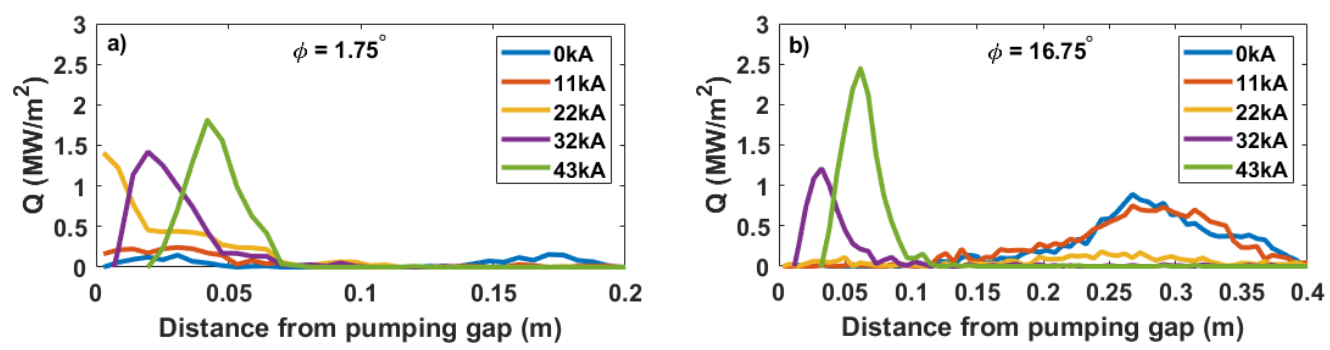

FIG. 5. Profiles of experimental heat flux at a) $\varphi=1.75^{\circ}$ and b) $\varphi=16.75^{\circ}$ corresponding to the mimic configurations shown in Fig. 4. 

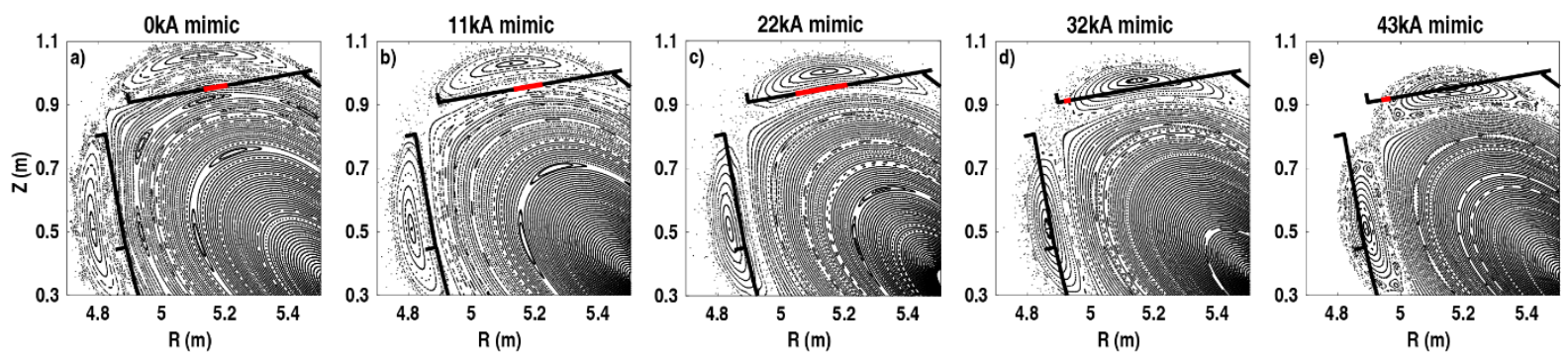

FIG. 6. Poincare plots at $\varphi=16.75^{\circ}$ for the a) $0 \mathrm{kA}$, b) $11 \mathrm{kA}$, c) $22 \mathrm{kA}$, d) $32 \mathrm{kA}$, and e) $43 \mathrm{kA}$ mimic configurations. Red lines indicate position on horizontal target (defined by half-width) of experimental heat flux.

\section{COMPARISON TO FIELD LINE DIFFUSION CALCULATIONS}

The good correspondence between the measured heat fluxes and the Poincare plots indicate that the W7$\mathrm{X}$ magnetic field is reasonably close to the ideal (no error field) stellarator symmetric magnetic field topology $[21,22]$. This correspondence can be further explored by comparing the fluxes to a simple field line diffusion model as implemented in the DIV3D code [1], which was used to design the scraper element and is similar to methods used to design the other W7-X divertor components. In the DIV3D model, field lines are traced initiating randomly from a closed flux surface inside the LCFS using a provided magnetic field description. At each step $\Delta_{\|}$along the line when integrating the field line differential equations, a cross-field displacement $\Delta_{\perp}$ with random azimuthal angle is applied corresponding to a 'magnetic diffusivity' $D_{m}=\Delta_{\perp}^{2} / \Delta_{\|}$. Each field line is assigned an equal fraction of the power crossing the closed surface and followed forwards and backwards with diffusion until it intersects a plasma facing component. The component is subdivided into the largest possible triangular facets while maintaining the desired geometric fidelity to a have a high computational efficiency. A heat flux is calculated by further dividing the components into triangular or rectangular regions and dividing the number of intersections by the area. This model assumes there are no parallel temperature gradients or heat flux mitigation from volumetric losses such as radiation. The magnetic diffusivity can be related to plasma parameters as $\mathrm{D}_{\mathrm{m}}=\mathrm{D} \perp / \mathrm{v}$ th, with $\mathrm{v}_{\text {th }}$ the thermal velocity. The value used when designing the scraper element is $D_{m}=3.155 \cdot 10^{-6} \mathrm{~m}^{2} / \mathrm{m}$, which could correspond to $\mathrm{D}_{\perp}=3 \mathrm{~m}^{2} / \mathrm{s}$ and $T_{\mathrm{e}}=5 \mathrm{eV}$, although caution should be used when assigning physical significance to these values. The design point was chosen to reproduce measured heat flux widths from the W7-AS stellarator and to match the assumptions made during the design process of the other divertor components.

Comparison of the fluxes from DIV3D to experimental data can be used to determine the best diffusivity to make predictions for OP1.2 and OP2 scenarios and determine if a simple diffusive model is sufficient or if higher fidelity simulations are required. Figure 7 shows heat fluxes calculated using DIV3D with $2 \mathrm{MW}$ of input power for three values of magnetic diffusivity in the $43 \mathrm{kA}$ (a-c) and 22kA (d-f) mimic configurations. Qualitatively, the patterns are similar, however some differences can be observed from the images. The

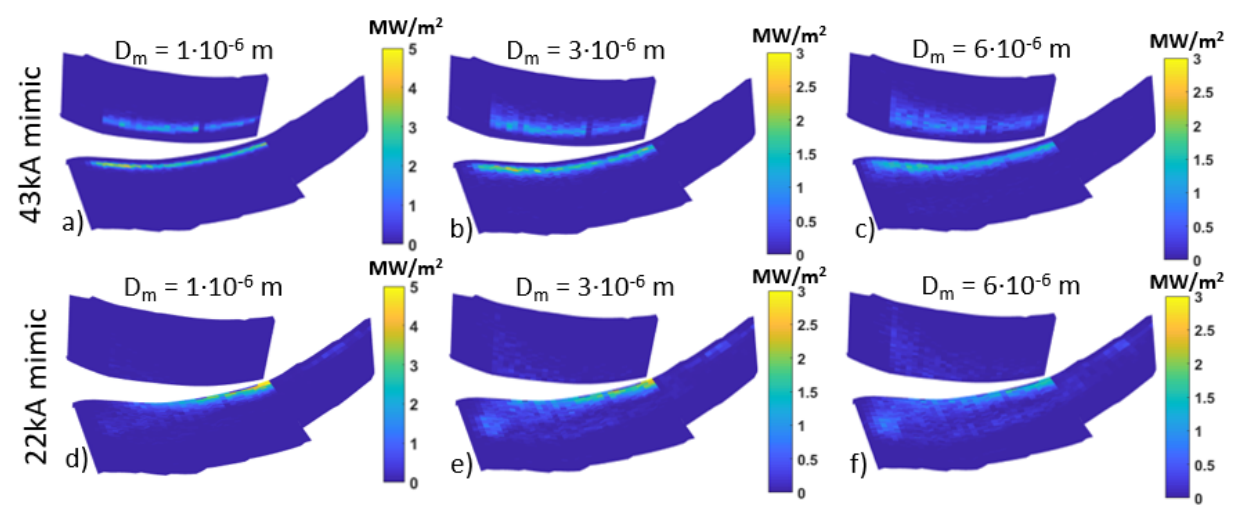

FIG. 7. Heat fluxes calculated by DIV3D for $2 M W$ of input power for the $43 \mathrm{kA}(\mathrm{a}-\mathrm{c})$ and $22 \mathrm{kA}$ mimic configuration for (a,d) $D_{m}=1 \cdot 10^{-6} \mathrm{~m}^{2} / \mathrm{m}$, (b,e) $D_{m}=3 \cdot 10^{-6} \mathrm{~m}^{2} / \mathrm{m}$, and $(c, f) D_{m}=6 \cdot 10^{-6} \mathrm{~m}^{2} / \mathrm{m}$. 
additional 'stripe' seen in the experimental data along the lowloaded divertor component for the 43kA configuration (Fig. 4e) is not observed in the DIV3D simulations (Fig. 7a-c). The peak flux in the $22 \mathrm{kA}$ configuration is closer to the edge in the experimental data than in DIV3D.

The heat fluxes on the horizontal target at $\varphi=1.75^{\circ}$ from experiment and DIV3D for several values of $D_{m}$ are shown in Fig. 8. In the 0kA configuration the heat flux peak is due to the limiting of the closed flux surfaces, and of a low magnitude as the target plate extends deeper inside the LCFS at higher toroidal angles (near $16.75^{\circ}$ ). The simulated fluxes show only a weak dependence on $D_{m}$ as the parallel connection lengths are low. In the $22 \mathrm{kA}-43 \mathrm{kA}$ configurations the simulated heat flux shows the expected trends for a scrape off layer plasma intersecting a plate. As the cross-field diffusion increases, the peak heat flux decreases and the wetted area (or profile width) increases. The $11 \mathrm{kA}$ configuration is in a marginal state between limited and island-diverted. In all cases the peak heat flux is close to or lower than the simulated value at the design point $\left(\mathrm{D}_{\mathrm{m}} \approx 3 \cdot 10^{-6} \mathrm{~m}^{2} / \mathrm{m}\right)$, as expected due to the neglect of dissipation. The heat flux profiles at $\varphi=16.75^{\circ}$ are shown in Fig. 9. At this toroidal position the $0 \mathrm{kA}$ and $11 \mathrm{kA}$ configurations are limited, while the $32 \mathrm{kA}$ and $43 \mathrm{kA}$ configurations are diverted. A special case is observed in the $22 \mathrm{kA}$ configuration, where the peak flux increases with increasing cross-field diffusion. This occurs because this region is only in close proximity to the LCFS, and sufficient cross-field diffusion is required to wet it, see Fig. 6c.

The poloidal position of the experimental flux at $\varphi=16.75^{\circ}$ agrees fairly well with DIV3D, however at $\varphi=1.75^{\circ}$ the experimental flux is shifted $1-3 \mathrm{~cm}$ towards the pumping gap. This is particularly evident in the $22 \mathrm{kA}$ comparison (Fig. $8 \mathrm{c}$ ), where the experimental flux increases monotonically to the pumping gap, while the DIV3D fluxes begin to decrease near the gap. This is potentially dangerous, as the DIV3D calculations already predict significant fluxes onto the target sides inside the pumping gap and a further shift in this direction could cause loading on low-rated components in the gap. In fact, some melting of stainless components in the pumping gap was observed after the campaign, which possibly occurred during these experiments. Across the comparison no single value of $\mathrm{D}_{\mathrm{m}}$ reproduces the magnitude and width in all cases, however $\mathrm{D}_{\mathrm{m}} \sim 2 \cdot 10^{-6} \mathrm{~m}^{2} / \mathrm{m}$ provides a conservative estimate with reasonable agreement with both magnitude and position of heat flux peaks, particularly when considering the fidelity of the model and the low computational requirements. A conservative calculation is preferred to incorporate a safety margin into the design process.
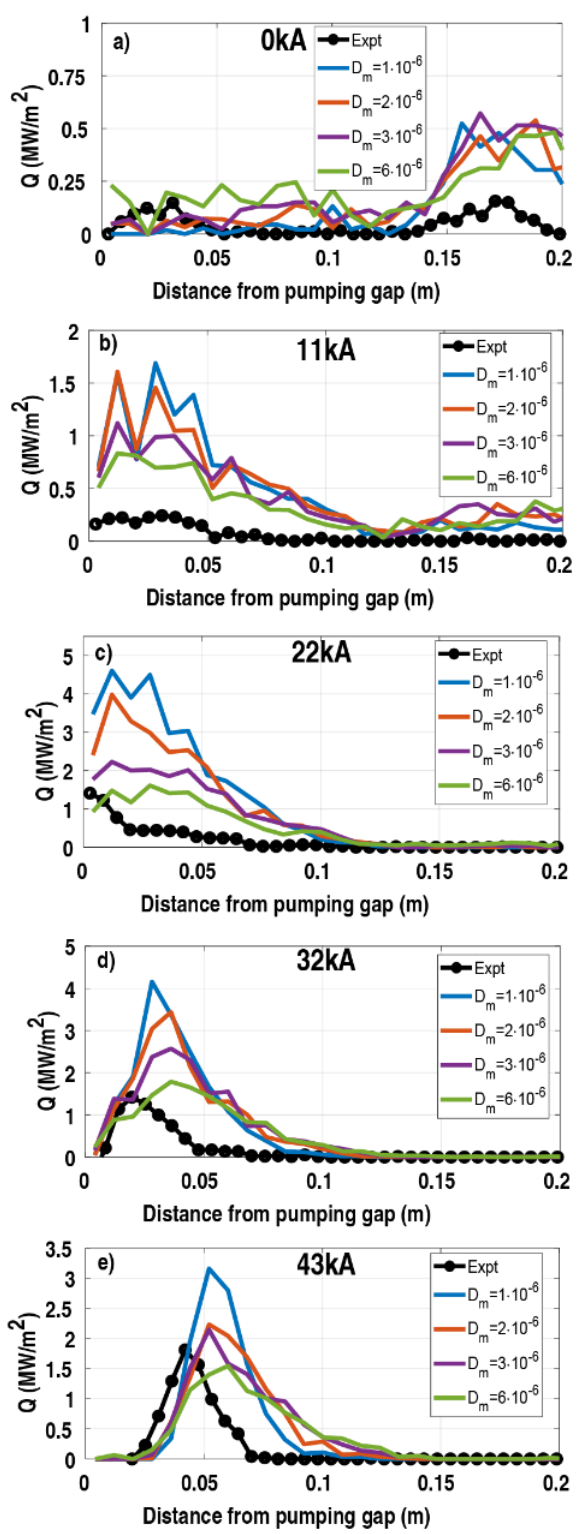

FIG. 8. Heat fluxes calculated from experiment (black symbols) and DIV3D for $2 M W$ of input power and (blue) $D_{m}=1 \cdot 10^{-6}$ $\mathrm{m}^{2} / \mathrm{m}$, (orange) $D_{m}=2 \cdot 10^{-6} \mathrm{~m}^{2} / \mathrm{m}$, (purple) $D_{m}=3 \cdot 10^{-6} \mathrm{~m}^{2} / \mathrm{m}$ and (green) $D_{m}=6 \cdot 10^{-6} \mathrm{~m}^{2} / \mathrm{m}$ in the (a) $0 \mathrm{kA}$, (b) $11 \mathrm{kA}$, (c) $22 \mathrm{kA}$, (d) $32 \mathrm{kA}$, and (e) $43 \mathrm{kA}$ configurations on the horizontal target at $\varphi=1.75^{\circ}$.

\section{ANALYSIS OF EFFECTS LEADING TO POLOIDAL SHIFT IN HEAT FLUX PROFILES}

The poloidal misalignment of the experimental and simulated profiles could be due to a number of factors, including vacuum error fields [22,23], divertor misalignment, low geometric fidelity in the diffusion simulations, plasma-induced changes to the configuration (finite beta and toroidal current), and transport effects such as drifts. To assess some of these effects, experimental heat flux profiles were processed from two additional cameras for the $22 \mathrm{kA}$ and $43 \mathrm{kA}$ configurations. Figure 10 shows heat flux profiles from upper module 4 (shown in the previous figures), and data from the two additional cameras at $\varphi=1.75^{\circ}$. The camera viewing the upper divertor module shows a shift away from the pumping gap for $1-2 \mathrm{~cm}$ in both configurations, potentially due to cross-field drifts which introduce an up-down asymmetry. Another camera viewing a lower 

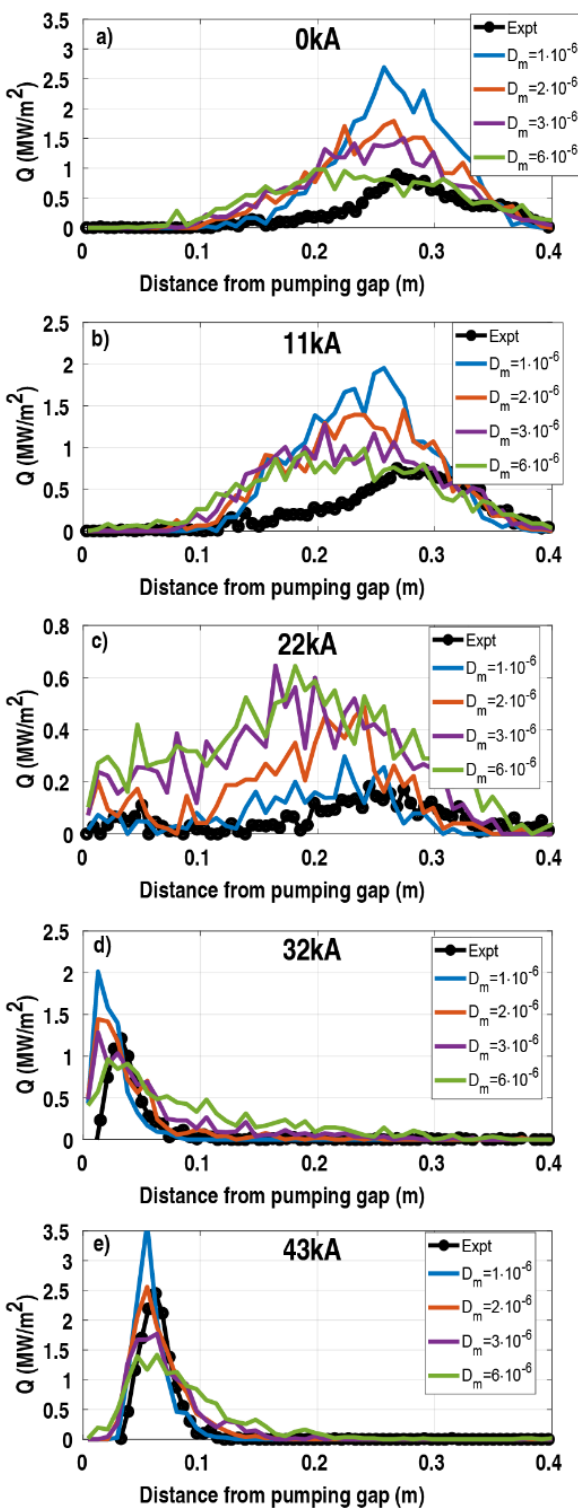

FIG. 9. Heat fluxes calculated from experiment (black symbols) and DIV3D for $2 \mathrm{MW}$ of input power and (blue) $D_{m}=1 \cdot 10^{-6} \mathrm{~m}^{2} / \mathrm{m}$, (orange) $D_{m}=2 \cdot 10^{-6} \mathrm{~m}^{2} / \mathrm{m}$, (purple) $D_{m}=3 \cdot 10^{-6} \mathrm{~m}^{2} / \mathrm{m}$ and (green) $D_{m}=6 \cdot 10^{-6} \mathrm{~m}^{2} / \mathrm{m}$ in the (a) $0 \mathrm{kA}$, (b) $11 \mathrm{kA}$, (c) $22 \mathrm{kA}$, (d) $32 \mathrm{kA}$, and (e) $43 \mathrm{kA}$ configurations on the horizontal target at $\varphi=16.75^{\circ}$
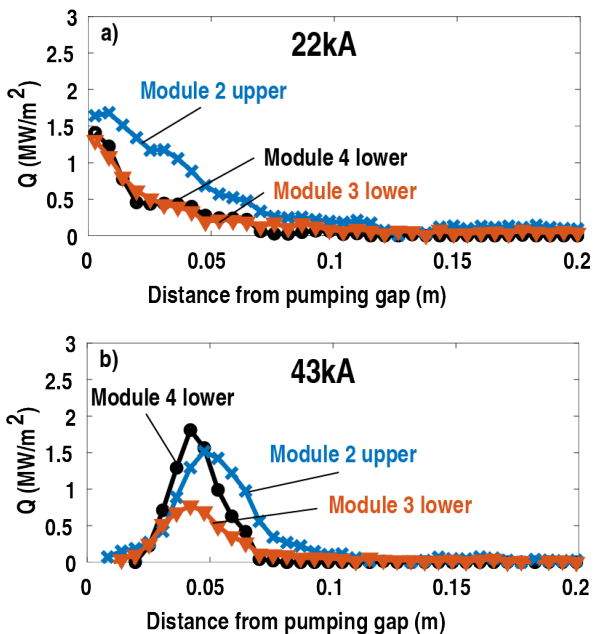

FIG. 10. Experimental heat fluxes from three cameras for the (a) $22 \mathrm{kA}$ and (b) $43 \mathrm{kA}$ configurations on the horizontal target at $\varphi=1.75^{\circ}$.

module shows a similar profile as upper module 4 in the $22 \mathrm{kA}$ configuration, with a factor difference of $\sim 2 \mathrm{x}$ in the magnitude in the $43 \mathrm{kA}$ configuration. A poloidal shift is not observed when comparing the two lower viewing cameras. These up-down and module to module variations include contributions from divertor misalignment, drifts, and error fields which are difficult to isolate.

The error fields were not corrected using the trim coils during the mimic experiments as there was insufficient experimental time to perform compass scans or flux surface mapping, although error fields are known to affect flux patterns in W7-X [24]. One effect of the measured error fields in $\mathrm{W} 7-\mathrm{X}$ is to reduce the rotational transform, in some cases by as much as $2 \%$ $[24,25]$. As the primary effect of the increasing toroidal current in the SE-RS is to increase the rotational transform profile, a shift in the peak heat flux towards the pumping gap could be caused by an error field induced transform reduction. This effect can be explored by adjusting the planar coils to reduce the transform profile. The relationship between transform and planar coil current is nearly linear, with a $2 \%$ reduction corresponding to a $\sim 1200 \mathrm{~A}$ increase in $\mathrm{I}_{\mathrm{A}}$ and an equal reduction in $\mathrm{I}_{\mathrm{B}}$. The heat fluxes for the $22 \mathrm{kA}$ and $43 \mathrm{kA}$ configuration with a $2 \%$ transform reduction are shown in Fig. 11 using $\mathrm{D}_{\mathrm{m}}=1 \cdot 10^{-6} \mathrm{~m}^{2} / \mathrm{m}$ (to clearly show the heat flux patterns). For example, in the 43kA configuration a 1200A change reduces the central vacuum transform from 0.840 to 0.823 , which shifts the heat flux peak $\sim 2-3 \mathrm{~cm}$ towards the pumping gap (compare Fig. 7a and Fig. 11b). This is the same effect as reducing the mimicked toroidal current, with $2 \%$ transform reduction corresponding to a decrease of $\sim 13-14 \mathrm{kA}$ in the mimicked toroidal current. For this reason, it is clear that a net transform reduction of this magnitude is not observed as the 22kA configuration would be in a limited shape (Fig. 12a), which is not supported by the heat flux data.

The inverse effect is observed in real time during the experiment. The pressure gradient drives a bootstrap current, and the net toroidal current increases on a relatively slow timescale ( $\sim$ seconds) depending on the internal inductance and resistivity of the plasma. This effect, and the resulting change in the magnetic 
a)

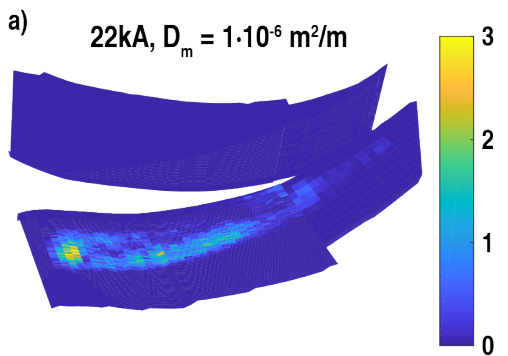

b)

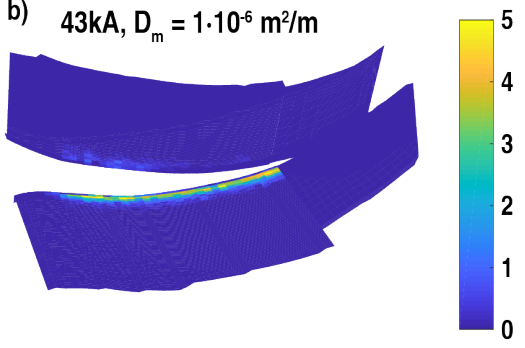

FIG. 11. Simulated heat flux patterns for the (a) $22 \mathrm{kA}$ and (b) $43 \mathrm{kA}$ configurations with the rotational tranform
reduced by $2 \%$.

topology is exactly that which causes the predicted overload scenario that the scraper element is designed to mitigate. Figure 12 shows the evolution of the experimental heat flux profiles and the net toroidal current for a 12 second discharge in the $43 \mathrm{kA}$ configuration with $2 \mathrm{MW}$ of input power. Over the discharge the toroidal current reaches $\sim 5 \mathrm{kA}$, resulting in a $1-2 \mathrm{~cm}$ shift of the heat flux profile away from the pumping gap. At $\varphi=16.75^{\circ}$ the magnitude of the heat flux peak increases with time, which has been speculated to be due to drift effects, although this requires further analysis. The observed currents of $\sim 5 \mathrm{kA}$, which have not reached steady state over 12 seconds, suggests that the toroidal current will reach values sufficient to cause substantial modifications to the heat fluxes in certain OP2 plasmas. If the predicted net toroidal currents of $\sim 43 \mathrm{kA}$ are obtained a mitigation strategy will likely be required.

\section{CONCLUSIONS}

Magnetic configurations developed to simulate magnetic topology changes corresponding to OP2 plasma scenarios were successfully employed to mimic these effects using the W7-X coil set. The plasma was adjusted from limited to island diverted over a series of discharges, including the $22 \mathrm{kA}$ mimic case with large heat fluxes at the edge of the horizontal target and into the pumping gap. Long discharges in the $43 \mathrm{kA}$ mimic configuration result in sufficient toroidal current to cause observable shifts in the heat flux profile. These results suggest that a similar evolution due to net toroidal current and beta in OP2 will result in unacceptable fluxes, requiring mitigation or avoidance. Similar experiments were recently performed in OP $1.2 \mathrm{~b}$ to confirm the predicted flux patterns onto two installed scraper elements [16], and the protection of the relevant divertor components.

The measured flux patterns are largely as expected from inspection of the field topology, e.g., using Poincare plots and field line tracing, indicating that the magnetic field is reasonably close to the ideal one. The flux patterns are approximately reproduced in magnitude and position from field line diffusion calculations using DIV3D for a range of diffusivities close to that used to design the scraper element. However, unexpected wetting of the low-loaded divertor component at $43 \mathrm{kA}$ and a poloidal shift towards the pumping gap for toroidal angles near $0^{\circ}$ are observed. In addition, a single value of magnetic diffusivity was not sufficient to describe the measured fluxes. Several potential sources of this discrepancy were explored, with an iota reduction due to error fields, observed differences in the camera data possibly due to drifts or divertor misalignment, and evolution of the toroidal current during the discharge all capable of producing a shift of several centimeters. Given the multiple and possibly counteracting sources of error it is difficult to identify a single cause with the data presently available. Further comparisons with higher fidelity simulations such as EMC3-EIRENE [27,28], and experimental scans of collisionality via power and density variation may inform the best diffusivity to use to model future W7-X experiments. EMC3-EIRENE simulations will also allow for a comparison with the particle fluxes, as inferred from filtered camera (e.g., H-alpha) measurements.
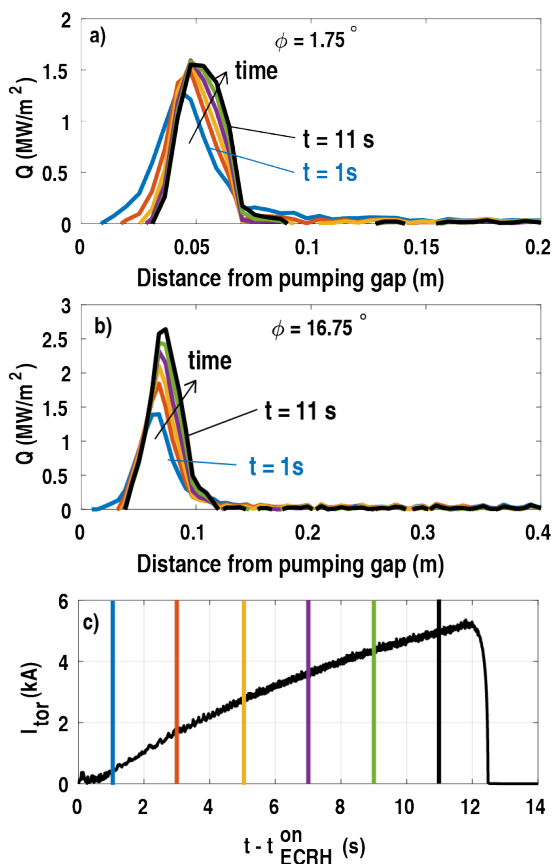

FIG. 12. Evolution of the a) heat flux profile at $\varphi=1.75^{\circ}$, b) heat flux profile at $\varphi=16.75^{\circ}$ and c) toroidal current during a

12 second discharge in the $43 \mathrm{kA}$ configuration. 


\section{ACKNOWLEDGEMENTS}

This material is based upon work supported by the US Department of Energy under contract number DE-AC0500OR22725. This work has been carried out within the framework of the EUROfusion Consortium and has received funding from the Euratom research and training programme 2014-2018 under grant agreement No 633053. The views and opinions expressed herein do not necessarily reflect those of the European Commission.

\section{REFERENCES}

[1] J.D. Lore, et al., Design and analysis of divertor scraper elements for the W7-X stellarator, IEEE TPS 42, (2014) 539.

[2] J.D. Lore, et al., Modeling and preparation for experimental testing of heat fluxes on W7-X divertor scraper elements, IEEE TPS 46, 1387 (2018).

[3] J. Fellinger, et al., Integration of uncooled scraper elements and its diagnostics into Wendelstein 7-X, Fusion Eng. Des. 24, 226 (2017).

[4] J. Geiger, et al., Effects of net currents on the magnetic configuration of W7-X, Contrib. Plasma Phys. 50 (2010), 770.

[5] J. Boscary et al., Design improvement of the target elements of the Wendelstein 7-X divertor, Fusion Eng. Des. 87, 1453 (2012).

[6] Y. Gao, et al., Effects of toroidal plasma currents on the strike-line movements on W7-X, proceedings of the $45^{\text {th }}$ EPS Conference on Plasma Physics, (2018), O3.104.

[7] A. Loarte, et al., Effects of divertor geometry on tokamak plasmas, Plasma Phys Control Fusion 43, R183 (2001).

[8] H. Peraza-Rodriguez, et al., Bootstrap current control studies in the Wendelstein 7-X stellarator using the free-plasmaboundary version of the SIESTA MHD equilibrium code, Plasma Phys. Control. Fusion 60 (2018) 025023.

[9] Y. Turkin, et al., Current control by ECCD for W7-X, Fusion Sci. Technol., 50 (3), 387, (2006)

[10] A. Lumsdaine, et al., Modeling and analysis of the W7-X high heat-flux divertor scraper element, IEEE TPS 42 (2014) 545

[11] R. Maingi, et al., Pump plenum pressure dependence on divertor plasma parameters and magnetic geometry in the DIII-D tokamak, Nucl. Fusion 39 (1999) 1187

[12] H. Hoelbe, Control of the magnetic topology and plasma exhaust in the edge region of Wendelstein 7-X: A numerical study, IPP report 18/4, February 2016.

[13] H. Hoelbe, et al, Access to edge scenarios for testing a scraper element in early operation phases of Wendelstein 7-X, Nucl. Fusion 56 (2016) 025015.

[14] S.P. Hirshman, et al., Steepest-descent moment method for three-dimensional magnetohydrodynamic equilibria, Phys. Fluids 26 (1983) 3553.

[15] S.P. Hirshman, et al., Three-dimensional free boundary calculations using a spectral Green's function method, Comput. Phys. Commun, 43 (1986) 143.

[16] M. Drevlak, et al., PIES free boundary stellarator equilibria with improved initial conditions, Nucl. Fusion 45 (2005) 731.

[17] M. Jakubowski, et al., Infrared imaging systems for wall protection in the W7-X stellarator, Rev. Sci. Inst, 89, $10 \mathrm{E} 116$ (2018).

[18] F. Pisano, et al., Towards a new image processing system at Wendelstein 7-X: From spatial calibration to characterization of thermal events, Rev. Sci. Inst., 89, 123503 (2018).

[19] Puig Sitjes, A., et al., Wendelstein 7-X Near Real-Time Image Diagnostic System for Plasma-Facing Components Protection, Fusion Science and Technology 74, 116 (2017)

[20] Ali. A., et al., Experimental results of near real-time protection system for plasma facing components in Wendelstein 7$X$ at GLADIS, Physica Scripta 2017, 014074 (2017)

[21] T.S. Pedersen, et al., Confirmation of the topology of the Wendelstein 7-X magnetic field to better than 1:100,000, Nature Communications 7 (2016) 13493.

[22] S. Bozhenkov, et al., Measurements and correction of the 1/1 error field in Wendelstein 7-X, Nuclear Fusion 59, 026004 (2018).

[23] S.A. Bozhenkov, et al., Methods for measuring 1/1 error field in Wendelstein 7-X stellarator, Nuclear Fusion 56, 076002 (2016).

[24] S.A. Lazerson, et al., Error fields in the Wendelstein 7-X stellarator, Plasma Phys and Control Fusion 60, 124002 
[25] M. Otte, et al., Setup and initial results from the magnetic flux surface diagnostics at Wendelstein 7-X, Plasma Phys Control Fusion 58, 064003 (2016)

[26] G.A. Wurden, et al, A divertor scraper observation system for the Wendelstein 7-X stellarator, Rev. Sci. Inst. 89 (2018) 10E102.

[27] Y. Feng, et al., A 3D Monte Carlo code for plasma transport in island divertors, J. Nucl. Mater., 241-243, 930 (1997)

[28] Y. Feng, et al., Recent improvements in the EMC3-Eirene code, Contrib. Plasma Phys. 54 (2014) 426 